\title{
Screening of women with aesthetic prostheses in dedicated sessions of a population-based breast cancer screening programme
}

\author{
Silvia Deandrea ${ }^{1} \cdot$ Laura Cavazzana $^{2} \cdot$ Niccolò Principi $^{2} \cdot$ Ester Luconi $^{3} \cdot$ Mauro Campoleoni $^{4}$. \\ Anan Judina Bastiampillai ${ }^{1} \cdot$ Lucia Bracchi $^{4} \cdot$ Lauro Bucchi $^{5}$. Stella Pedilarco ${ }^{6}$. Antonio Piscitelli ${ }^{2}$. \\ Maria Silvia Sfondrini ${ }^{6} \cdot$ Anna Rita Silvestri $^{1} \cdot$ Silvana Castaldi $^{7,8}$
}

Received: 10 October 2020 / Accepted: 14 April 2021 / Published online: 5 May 2021

(c) The Author(s) 2021

\begin{abstract}
Background Women with aesthetic prostheses must be included in the target population of mammography screening programmes. Breast implants are radiopaque and partially obscure the breast tissue. This can be avoided with the use of the Eklund technique, which causes an increased radiation exposure. In this study, augmented women undergoing a dedicated protocol within a population-based screening programme were compared according to selected indicators with the standard screening population. Essential dosimetric parameters and their time trend were also assessed.

Materials and methods The study was conducted in a screening centre in Milan in the years 2009-2016. The screening protocol for women with breast implants included a double-read mammography with the Eklund views, ultrasound and clinical breast examination.

Results A total of 28,794 women were enrolled, including 588 (2\%) women with breast implants and 28,206 (98\%) undergoing the standard screening protocol. The invasive assessment rate was $9.0 \%$ for women with breast implants vs. $15 \%$ in the standard cohort. The surgical referral rate was $2.2 \%$ vs. $0.9 \%$. The detection rate was similar in the two groups $(4.0$ and $4.5 \%$, respectively). There were significant differences in the average glandular dose according to the mammography equipment. The use of the Eklund views increased over time.

Conclusions Screening of augmented women according to a specific protocol in the contexts of population-based programmes is feasible. Observed differences in screening indicators relative to the standard screening population require further research. The increasing use of Eklund views probably results from quality assurance measures associated with screening programmes.
\end{abstract}

Keywords Breast implants · Breast neoplasms · Diagnostic screening programmes $\cdot$ Mammography

\begin{tabular}{ll}
\multicolumn{2}{l}{ Abbreviations } \\
AGD & Average glandular dose \\
BI-RADS & Breast Imaging-Reporting and Data System \\
BSP & Breast cancer screening programme \\
BT & Breast thickness \\
CBE & Clinical breast examination
\end{tabular}

Laura Cavazzana

laura.cavazzana@unimi.it

1 Health Protection Agency Metropolitan City of Milan, Milan, Italy

2 Post Graduate School of Public Health, University of Milan, Milan, Italy

3 Fondazione IRCCS Ca' Granda Ospedale Maggiore Policlinico, Milan, Italy

4 Medical Physics Unit, Fondazione IRCCS Ca' Granda Ospedale Maggiore Policlinico, Milan, Italy

$\begin{array}{ll}\text { CI } & \text { Confidence interval } \\ \text { DR } & \text { Detection rate } \\ \text { EUREF } & \begin{array}{l}\text { European Reference Organisation for Qual- } \\ \text { ity Assured Breast Screening and Diagnostic } \\ \end{array} \\ & \text { Services } \\ \text { FNA } & \text { Fine needle aspiration }\end{array}$

5 Romagna Cancer Registry, Romagna Cancer Institute (IRCCS Istituto Romagnolo per lo Studio dei Tumori, IRST, "Dino Amadori"), Meldola, Forlì, Italy

6 Division of Radiology, Fondazione IRCCS Ca' Granda Ospedale Maggiore Policlinico, Milan, Italy

7 Department of Biomedical Sciences for Health, University of Milan, Milan, Italy

8 Quality Unit, Fondazione IRCCS Ca' Granda Ospedale Maggiore Policlinico, Milan, Italy 


$\begin{array}{ll}\text { kV } & \text { Kilovoltage } \\ \text { LCC } & \text { Left craniocaudal } \\ \text { LMLO } & \text { Left mediolateral oblique } \\ \text { mAS } & \text { Milliamperesecond } \\ \text { NCB } & \text { Needle core biopsy } \\ \text { OR } & \text { Odds ratio } \\ \text { RCC } & \text { Right craniocaudal } \\ \text { RMLO } & \text { Right mediolateral oblique } \\ \text { US } & \text { Ultrasound }\end{array}$

\section{Introduction}

Breast augmentation is one of the most frequently performed aesthetic surgeries among women. Approximately, 5 to 10 million women worldwide have breast implants [1]. The American Society of Aesthetic Plastic Surgeons has reported an increase of 207\% from 1997 to 2016, showing an exponential growth in recent years [2]. In Italy, according to data for 2017 , nearly 50,000 breast augmentation surgeries are done annually [3].

Over the last years, the scientific community has raised the hypothesis that breast implants might be related to an increase in the incidence of breast cancer. In fact, many studies [4-6] have shown that implants have no effects on the risk of disease, although an increase in the incidence of the rare implant-associated anaplastic large cell lymphoma has been reported [7].

A different concern has arisen from the fact that breast implants are radiopaque and, in part, obscure the breast tissue. Some studies have highlighted that the prosthesis and its location could make early cancer detection by mammography more challenging, eventually leading to delay in diagnosis [8-16]. In spite of that, mammography remains an accurate method of early detection of breast cancer in women with prostheses [17, 18]. For this reason, the 2006 European guidelines for quality assurance in breast cancer screening and diagnosis recommend that breast cancer screening programmes (BSP) offer mammography to all women in the target age range, including those with aesthetic prosthesis [19]. Notably, however, the European guidelines recommended that these women should be screened in clinics where ultrasound (US) is available and that radiographers should receive specific training.

Indeed, the physical presence of an implant may hamper the application of standard radiographic procedures and stresses the need for a specific management protocol, including special views with back placement of the implant (Eklund views), and a specific technical expertise of the radiographer [20]. Eklund views are performed by displacing the implant posteriorly against the chest wall, pulling the breast tissue over and in front of the implant. This technique allows better visualization of the glandular tissue, in particular for craniocaudal views [21,22]. On the other hand, when additional views are needed in order to perform the Eklund technique, women are exposed to a greater dose of radiation $[11,23]$. The factors that affect the absorbed radiation dose can be summarized in the thickness of the tissue analysed, the amount of compression used, and the number of views taken.

In 2009, in order to provide women with aesthetic breast prostheses with an appropriate management within the local population-based BSP, the Milan Local Health Authority implemented a specific screening protocol in a reference centre, operating in one of the screening centres. This study is the first Italian study to describe the management of these women within a population-based BSP. Its objective is to report (1) the main screening and management indicators in comparison with nonaugmented women undergoing standard screening in the same period, and (2) the main dosimetric parameters and their trend over time.

\section{Materials and methods}

\section{Setting}

In the city of Milan (about 1.300.000 inhabitants), the BSP for women aged 50-69 (approximately 200,000 for each screening round) was started in 1999-2000. The process to extend the target age to 45-74 was undertaken in 2016, although women aged 45-49 and 70-74 had already been screened upon request. The interval is biennial for women aged 50-74 and annual for women aged 45-49. Standard indicators are regularly provided to the Department of Health of the Lombardy regional Administration [24] and the National Centre for Screening Monitoring [25]. The average response rate is $67 \%$ and the recall rate is $10 \%$ for the first screens and $4 \%$ for subsequent ones.

Between 2009 and 2016, the reference centre for the screening of women with breast implants has been at the Breast Radiology Unit of the Fondazione IRCCS Ca' Granda Ospedale Maggiore Policlinico (henceforth "Policlinico"), a public teaching and research hospital located in the centre of Milan. The present study focuses on this period. From 2017 onwards, due to a re-organization of healthcare services following the Lombardy Region healthcare reform [26], screening women with breast implants is no longer the responsibility of the BSP, although the Unit still provide early diagnosis for physician-referred and self-referred women with breast implants. The Breast Radiology Unit of the Policlinico is a hub for the diagnosis and treatment of breast diseases (with about 33,000 exams per year). It performs approximately $12 \%$ of screening mammographies and further assessment tests for the BSP covering the area of Milan [27, 28]. 


\section{Standard screening protocol}

During the study period, all women eligible for the BSP of the Milan Local Health Authority received a personal appointment letter. Self-referral of eligible women was also accepted. The screening mammography and the diagnostic assessment tests were provided by centres established in hospital-based radiology services. All women had a two-view digital mammography with independent double reading. Selected early recall (assessment after 6 months) and early rescreening (screening mammography after 1 year) policies were practiced. Women with positive results (BI-RADS 3-5) were contacted by telephone and referred for diagnostic assessment, which included supplementary mammography, US, clinical breast examination (CBE), and cytological and histological invasive procedures-if appropriate.

\section{Screening of women with breast prostheses}

The screening invitation letters included a sentence asking women with aesthetic prostheses to contact the BSP communication centre. For women adhering to this recommendation, a specialized appointment was set in the Breast Radiology Unit of the Policlinico. The Unit was responsible both for the screening of women and their diagnostic workup. These were done in dedicated screening sessions (also referred to as prosthesis pathway) and included a mammography performed by a trained technician and double-read, with the Eklund technique in the craniocaudal views, and US and CBE performed by a radiologist. In the case of a negative result, women were re-invited at 1-year or 2-year intervals depending on the clinical opinion. In the case of a suspicious result, women underwent the same assessment tests as those offered to standard screenees.

\section{Mammography systems}

Up to January 2015, women were screened with two firstgeneration full field digital mammography systems, i.e. a General Electric Senograph 2000D (henceforth "2000D”) and a General Electric Senograph Essential (henceforth "ES"). From January 2015 on, women have been screened with a more recent Hologic Selenia Dimensions. This new equipment is provided with a specific post-processing software which improves the image quality in the presence of an implant. All equipments underwent a quality control programme for the assessment of the physical and technical requirements according to the EUREF protocols [29].

\section{Study population}

This is a retrospective cohort study. The cohort included all carriers of sub-glandular or retro-glandular aesthetic breast implants who entered the prosthesis pathway between November 2009 and July 2016.

The cohort of women undergoing the standard protocol of the BSP at the Policlinico during the same time period was used as a control group. Inclusion criteria were female sex, age between 45 and 74, and no previous history of breast cancer.

\section{Data collection}

For women undergoing the standard protocol of the BSP, data on basic screening and assessment of positive findings were real-time recorded during the daily operation. Histological information for screen-detected lesions was collected via search in the regional archive of the Hospital Discharge Records and in the databases of Pathology Departments at the surgical referral hospitals.

For the cohort of women with breast implants, information was collected from the Cancer Registry of the Agency for Health Protection of Milan (formerly, Milan Local Health Authority). A dataset was created that included demographic variables (age, nationality and residence), screening history, screening and assessment tests (type, date, results), and screening episode result.

The collection of dosimetric parameters was performed on a representative sample of the total number of images (47.6\%). For each image, the following data were collected: average glandular dose (AGD), breast thickness (BT), Eklund's implant-displacement technique (yes/no) and exposure parameter $(\mathrm{kV}, \mathrm{mAs}$, anode-filter combination, and compression force).

\section{Definitions}

In this article, screening episode indicates each single participation by all women to the biennial screening. The term session indicates a single time slot allocated for women within the screening episode. Screening round (first, subsequent) indicates the first participation and the subsequent ones.

\section{Data analysis}

Women with and without breast implants were compared according to age, year of mammography, nationality, screening round, and number of screening episodes using the chisquare test. Age was classified in five-year intervals and the year of mammography in two-year intervals. Univariate logistic regression models were built to test for the presence of a significant association between the presence of 
a breast implant and the age class (reference, 48-49), year of mammography (reference, 2009-2010), and nationality (reference, other, i.e. not Italian).

Assessment tests were further classified into non-invasive (mammography, CBE, US, and MRI) and invasive (needle core biopsy (NCB) and fine needle aspiration (FNA)). Rates, and $95 \%$ confidence intervals $(\mathrm{CI})$, were computed for invasive assessments, surgical referral (including both surgical biopsy and surgical intervention), early recall/rescreen and detection of cancer (or detection rate (DR) per 1000 women). Data are presented for women with breast implants and for the standard screening population, which considers all women screened and those referred for assessment separately. Due to the possibility of sending augmented women to a 1-year appointment, the 6-month recall was considered for the early recall/rescreen rate computation for the standard screening cohorts only.

Women with breast implants who underwent the whole dedicated screening procedure (mammography, US and CBE) were defined as screened per protocol. With the aim of disentangling the effect of a "per protocol" procedure and that of being a prosthesis carrier, we matched cases (augmented women) and controls from the standard screening population according to age interval, screening round and per-protocol status. The odds ratio (OR) for surgical referral was calculated from the contingency table (breast implant and surgical referral). For invasive examinations, the analysis was not performed, as there were no "per protocol" matched women without breast implants.

In order to determine whether the sample subjects with dosimetric information differed from those with no information in terms of distribution by age class, year of mammography, nationality and screening round, a two-sided Fisher exact test was performed. The level of statistical significance was set at $P<0.05$. The sample was demonstrated to be representative of the population of women with breast implants.

For all the views (right craniocaudal, RCC, left craniocaudal, LCC, right mediolateral oblique, RMLO and left mediolateral oblique, LMLO), the mean and median AGD and BT and their range were calculated for the whole sample, for different mammography systems (2000D vs. ES) and for the application of the Eklund technique (yes vs. no). $\mathrm{T}$ tests were used to evaluate the significance of the difference between mean AGDs. Each view was independently considered.

Finally, the significance of the increasing use of the Eklund procedures over time, using the semester as a time unit, was tested with an Armitage test versus the null hypothesis on no linear trend.

The management of data sets was done with the program KNIME Analytics Platform ver. 3.6.2 [30]. Statistical analyses were performed using the program " $\mathrm{R}$ " ver.3.5.1 (2018) [31].

\section{Results}

\section{Patient characteristics}

Between November 2009 and July 2016, 28,794 women participated in the BSP at the Breast Radiology Unit of the Policlinico. As shown in the upper row of Table 1, there were 588 (2\%) women with breast prostheses and $28,206(98 \%)$ women who were screened according to the standard protocol.

Table 1 shows the distribution of women in both cohorts according to age, year of mammography, nationality, screening round, and number of screening episodes, as well as the number of breast cancers detected. There was evidence for an inverse association between the age class and being a breast implant carrier. Augmented women had more often a single screening episode. Their different distribution by year of mammography reflected the gradual roll-out of the dedicated screening programme. Nationality and the number of screening rounds were not associated with the odds of a woman having a breast implant.

The OR from multiple regression analysis, shown in Table 2, was 1.44 (95\% C.I.: 1.16-1.79) for the age class 65-69, and 1.81 (95\% C.I.: 1.47-2.25) for the age class 70-74 as compared with the class 48-54. For the remaining classes, the OR was not significantly different from the unity.

\section{Screening and management indicators}

As shown in the upper row of Table 3, the total number of screening episodes was 67,991 , including 61,608 screening mammography sessions, 5384 assessment sessions and 999 prostheses protocol sessions. Table 3 also gives the number of tests and the indicators for women with breast implants, for women without breast implant (standard population) and for women without breast implant undergoing assessment only. Most women (87.3\%) were screened as per protocol, whilst for others one or more of the three tests of the protocol were not performed. Invasive assessment rate was $9.0 \%$ o for women with breast implants. The corresponding figure for women undergoing screening mammography was $15.2 \%$, and for women undergoing assessment $174.8 \%$. Surgical referral rate was $2.2 \%$ for women with breast implants, $0.9 \%$ for women undergoing screening mammography and $9.7 \%$ for women undergoing assessment. Finally, the DR was $4.0 \%$ in the augmented group and $4.5 \%$ in the control group.

When matched with nonaugmented women according to age interval, screening round and non-invasive tests performed as "per protocol", breast implant carriers showed a 
Table 1 Women's demographic and clinical characteristics at first observation in the study

\begin{tabular}{|c|c|c|c|}
\hline Characteristics & Women with breast implants $\mathrm{n}(\%)$ & Women without breast implants $\mathrm{n}(\%)$ & $\chi^{2}$ test (likelihood) ${ }^{\mathrm{a}}$ \\
\hline Number & $588(2.0)$ & $28,206(98.0)$ & \\
\hline \multicolumn{4}{|l|}{ Age class } \\
\hline $48-49$ & $5(0.9)$ & $151(0.5)$ & $p<0.001$ \\
\hline $50-54$ & $272(46.3)$ & $6479(23.0)$ & \\
\hline $55-59$ & $167(28.4)$ & $4478(15.9)$ & \\
\hline $60-64$ & $69(11.7)$ & $5317(18.9)$ & \\
\hline $65-69$ & $58(9.9)$ & $6177(21.9)$ & \\
\hline $70-74$ & $17(2.9)$ & $5604(19.9)$ & \\
\hline Mean age & $57.15($ sd 5,$10 ;$ range $49-74)$ & 62.16 (ds 7,03; range 48-74) & \\
\hline Year of mammography & & & $p<0.001$ \\
\hline 2009-2010 & $60(10.2)$ & $9560(33.9)$ & \\
\hline 2011-2012 & $249(42.4)$ & $9906(35.1)$ & \\
\hline 2013-2014 & $262(44.6)$ & $4210(14.9)$ & \\
\hline $2015-2016$ & $17(2.9)$ & $4530(16.1)$ & \\
\hline Nationality & $p=0.351$ & & \\
\hline Italian & $530(90.1)$ & $25,086(88.9)$ & \\
\hline Other & $58(9.9)$ & $3120(11.1)$ & \\
\hline Screening round & & & $p=0.764^{\mathrm{b}}$ \\
\hline First & $197(33.5)$ & $9284(32.9)$ & \\
\hline Subsequent & $391(66.5)$ & $18,922(67.1)$ & \\
\hline Number of screening episodes & & & $p<0.001^{\mathrm{c}}$ \\
\hline 1 & $278(47.3)$ & $9980(35.4)$ & \\
\hline 2 & $229(39.0)$ & $6001(21.3)$ & \\
\hline 3 & $68(11.6)$ & $9273(32.9)$ & \\
\hline$\geq 4$ & $13(2.2)$ & $2952(10.5)$ & \\
\hline Number of screen-detected cancers & 4 & 274 & - \\
\hline
\end{tabular}

${ }^{\mathrm{a}} p<0.05$ from $\chi^{2}$ test identifies a significant association between the explanatory variable (for example, age) and the response variable (breast implant or not breast implant). In particular, the $X^{2}$ from a likelihood compares the model only with an intercept and the model with the intercept and the variable

${ }^{b}$ the logistic regression for the chi square test was codified with the response variable as 1 in case of subsequent screening round and as 0 otherwise. The explanatory variable was codified as 1 in the presence of a breast implant and 0 otherwise

${ }^{c}$ Chi-square test: $p<0.05 \mathrm{H}_{0}$ : there is not an association between the presence of breast implant and the number of screening episodes. Ha: there is an association between the presence of breast implant and the number of screening episodes

Table 2 Odds Ratios from multiple regression analysis for age classes compared with the class $48-54$

\begin{tabular}{lll}
\hline Characteristic & $<55$ & $\begin{array}{l}\text { Odds Ratio (95\% } \\
\text { confidence inter- } \\
\text { val) }\end{array}$ \\
\hline Age class & Reference \\
\hline \multirow{3}{*}{$55-59$} & $1.07(0.85-1.35)$ \\
$60-64$ & $1.20(0.94-1.53)$ \\
$65-69$ & $1.44(1.16-1.79)$ \\
& $70-74$ & $1.81(1.47-2.25)$ \\
\hline
\end{tabular}

reduced risk to be referred to surgery $(\mathrm{OR}, 0.25 ; 95 \% \mathrm{CI}$, $0.14-0.43)$, suggesting a different management of these women not attributable to demographics or screening history characteristics.

\section{Dosimetric parameters}

In Table 4, the mean and the median for AGD and for BT for the whole sample, for different equipments and for the application of the Eklund technique are reported. As expected, the Eklund technique was usually not performed in MLO views, and compression was higher with the Eklund displacement. We observed a significantly $(p<0.001)$ lower AGD with the Selenia system vs. 2000D and ES for all the four views.

\section{Dosimetric parameters by Eklund technique}

In Table 5, AGD and BT for the different equipment are reported separately for Eklund technique (yes vs. No). When the technique was performed, AGD was confirmed to be 
Table 3 Screening and management indicators for women with breast implants and women without breast implant (all women with a screening mammogram and women undergoing assessment only)

\begin{tabular}{|c|c|c|c|}
\hline \multirow[t]{2}{*}{ Indicator } & \multirow{2}{*}{$\begin{array}{l}\text { Women with } \\
\text { breast implants }\end{array}$} & \multicolumn{2}{|c|}{ Standard screening population } \\
\hline & & $\begin{array}{l}\text { Women without breast } \\
\text { implants-all }\end{array}$ & $\begin{array}{l}\text { Women without breast } \\
\text { implants-assessment } \\
\text { only }\end{array}$ \\
\hline Screening episodes $(n)$ & 999 & 61,608 & 5384 \\
\hline Mammography $(n, \%)$ & $956(95.7)$ & $4269(6.4)$ & $4269(79.3)$ \\
\hline $\mathrm{CBE}(\mathrm{n}, \%)$ & 915 (91.6) & $4689(7.0)$ & $4689(87.1)$ \\
\hline US (n, \%) & 965 (96.6) & $4831(7.2)$ & $4831(89.7)$ \\
\hline MRI (n, \%) & $2(0.2)$ & $49(0.1)$ & $49(0.9)$ \\
\hline FNA $(\mathrm{n}, \%)$ & $0(0.0)$ & $33(0.1)$ & $33(0.6)$ \\
\hline NCB-VAB (n, \%) & $9(0.9)$ & $867(1.3)$ & $867(16.1)$ \\
\hline \multicolumn{4}{|l|}{$\begin{array}{l}\text { Invasive assessment, rate } \\
\quad(\%, 95 \% \mathrm{CIs})\end{array}$} \\
\hline FNA & 0 & $0.5(0.4-0.8)$ & $6.1(4.2-8.6)$ \\
\hline NCB-VAB & $9.0(4.1-17.0)$ & $14.7(13.8-15.7)$ & $168.7(158.7-178.9)$ \\
\hline Total & $9.0(4.1-17.0)$ & $15.2(14.2-16.5)$ & $174.8(162.9-187.5)$ \\
\hline Surgical referral rate $(\%, 95 \% \mathrm{CIs})$ & $2.2(1.4-3.3)$ & $0.9(0.8-0.9)$ & $9.7(9.0-10.6)$ \\
\hline $\begin{array}{l}\text { Early recall/rescreen rate }(\%, 95 \% \\
\text { CIs) }\end{array}$ & $2.4(1.4-3.3)$ & $0.9(0.8-1.0)$ & $10.0(9.2-10.8)$ \\
\hline Detection rate $(\% o, 95 \%$ CIs $)$ & $4.0(1.1-10.2)$ & $4.5(3.9-5.0)$ & - \\
\hline
\end{tabular}

$F N A$, Fine needle aspiration; $N C B-V A B$, Needle Core Biopsy/Vacuum Assisted Biopsy; $C I$, Confidence Interval lower with an automatic exposure control or an optimized exposure protocol (Selenia).

\section{Prevalence of the Eklund technique over time}

The prevalence for the Eklund technique over different periods was determined. There was a significant linear trend toward an increased implementation of the technique, from $45 \%$ in the first semester of activity to $83 \%$ in the last one (Armitage test, chi-squared test for trend, 43.59; degrees of freedom, $1 ; p$ value $<0.001$ ) [data not shown].

\section{Discussion}

\section{Principal findings}

The indicators studied provided evidence for a different management of augmented women compared with women undergoing the standard basic screening. Albeit similar to women in the screening cohort with respect to the risk of breast cancer, augmented women underwent the same tests as women referred for assessment because of suspicious mammograms. For this reason, neither of the two cohorts is an appropriate comparator for the augmented cohort. By implication, our results should mainly be used for healthcare planning purposes, that is, for assessing the potential workload and the investment needed in breast care services.
From this standpoint, our data suggest that augmented women screened according to this protocol do not require a higher volume of invasive tests than the standard screening population. The surgical referral rate, conversely, was higher. However, there was an inverse association between surgical referral and being a prosthesis carrier when women were matched with subjects undergoing the same protocol $($ mammogram $+\mathrm{US}+\mathrm{CBE})$. This result suggests a different management possibly according to clinical suspicion, to setting preference (inpatient surgical biopsy vs. outpatient), and to some unmeasured factors related to the status of prosthesis carrier.

The second key finding of the study is that the dosimetric parameters changed over time due to the upgrade to a more advanced technology (Selenia). In addition, radiographers have improved their capability in applying the Eklund technique as a first choice method for the craniocaudal views. This observation must be interpreted considering the advantage in terms of resources, staff education and professional improvement when cases requiring special skills are concentrated in a high-volume centre.

\section{Rationale issues}

The rationale of setting-up sessions of BSPs specifically dedicated to women with breast implants relies on the 2006 European guidelines for quality assurance in breast cancer screening and diagnosis. These, however, recommend that 
Table 4 Average glandular dose (AGD) and for BT (breast thickness) for the four views

\begin{tabular}{|c|c|c|c|c|c|c|c|c|}
\hline & \multicolumn{2}{|l|}{$\mathrm{RCC}$} & \multicolumn{2}{|l|}{ LCC } & \multicolumn{2}{|l|}{ RMLO } & \multicolumn{2}{|l|}{ LMLO } \\
\hline & AGD (mGy) & $\mathrm{BT}(\mathrm{mm})$ & AGD (mGy) & $\mathrm{BT}(\mathrm{mm})$ & AGD (mGy) & $\mathrm{BT}(\mathrm{mm})$ & AGD (mGy) & $\mathrm{BT}(\mathrm{mm})$ \\
\hline \multicolumn{9}{|l|}{ Total } \\
\hline Views & 452 & 473 & 450 & 469 & 397 & 473 & 390 & 469 \\
\hline Mean & 1.4 & 48.8 & 1.4 & 47.6 & 1.2 & 69 & 1.2 & 67.8 \\
\hline Median & 1.4 & 45.0 & 1.4 & 45.0 & 1.2 & 71 & 1.2 & 69 \\
\hline Range & $0.6-7.7$ & $18-108$ & $0.5-2.7$ & $20-127$ & $0.1-3.9$ & $29-110$ & $0.7-2.7$ & $31-118$ \\
\hline \multicolumn{9}{|l|}{ Systems } \\
\hline \multicolumn{9}{|l|}{ 2000D/ES } \\
\hline Views & 381 & 402 & 382 & 401 & 326 & 402 & 322 & 401 \\
\hline Mean & 1.5 & 48.8 & 1.5 & 47.9 & 1.3 & 67.2 & 1.3 & 66.1 \\
\hline Median & 1.4 & 45 & 1.5 & 45 & 1.2 & 69 & 1.2 & 67 \\
\hline Range & $0.7-7.7$ & $18-105$ & $0.7-2.7$ & $20-127$ & $0.6-2.2$ & $29-110$ & $0.7-2.7$ & $31-118$ \\
\hline \multicolumn{9}{|l|}{ Selenia } \\
\hline Views & 71 & 71 & 68 & 68 & 71 & 71 & 68 & 68 \\
\hline Mean & 1.1 & 48.6 & 0.9 & 46.1 & 1.1 & 78.9 & 1.1 & 78.3 \\
\hline Median & 0.9 & 43 & 0.8 & 41 & 1 & 78 & 1 & 77 \\
\hline Range & $0.6-6.2$ & 21-108 & $0.5-2$ & $23-100$ & $0.1-3.9$ & 53-106 & $0.8-1.6$ & $44-110$ \\
\hline \multicolumn{9}{|l|}{ Eklund } \\
\hline \multicolumn{9}{|l|}{ Yes } \\
\hline Views & 339 & 345 & 347 & 357 & 13 & 14 & 5 & 5 \\
\hline Mean & 1.4 & 43.3 & 1.4 & 42.6 & 1.5 & 47.1 & 1.6 & 47.8 \\
\hline Median & 1.5 & 45 & 1.5 & 45 & 1.5 & 45 & 1.4 & 45 \\
\hline Range & $0.6-4.8$ & $18-80$ & $0.5-2.7$ & $20-75$ & $1.1-2.2$ & $29-77$ & $1.3-2.4$ & $31-66$ \\
\hline \multicolumn{9}{|l|}{ No } \\
\hline Views & 113 & 128 & 102 & 111 & 384 & 459 & 385 & 464 \\
\hline Mean & 1.3 & 63.5 & 1.2 & 63.1 & 1.2 & 69.6 & 1.2 & 68.1 \\
\hline Median & 1.1 & 59 & 1.2 & 55 & 1.2 & 71 & 1.2 & 69 \\
\hline Range & $0.8-7.7$ & $37-108$ & $0.7-2.7$ & $31-127$ & $0.1-3.9$ & $41-110$ & $0.7-2.7$ & $31-118$ \\
\hline \multicolumn{9}{|c|}{ 2000D/ES vs. Selenia } \\
\hline$t$ test & -3.88 & - & & -14.04 & & & -3.55 & \\
\hline df & 80.01 & & & 93.25 & - & & 87.11 & - \\
\hline$p$ value & $<0.001$ & & & $p<0.001$ & & & $<0.001$ & \\
\hline $95 \% \mathrm{CI}$ & -0.57 to 0.18 & & & -0.70 to 0.53 & & & 0.28 to 0.28 & \\
\hline
\end{tabular}

RCC, right craniocaudal; LCC, left craniocaudal; RMLO, right medial-lateral oblique; LMLO, left medial-lateral oblique

mammographic imaging be performed in clinics where US is available and, more important, that radiographers be specifically trained and have up to date information and knowledge about breast implants [19]. It must be carefully considered that training and quality assurance are priorities in the implementation of BSPs.

Regarding the rationale for this study, two points need to be made. Firstly, the publication of our data was justified by the fact that the results of protocols for the management of women with breast implants attending BSP in Italy and elsewhere have never been reported in relevant publications.

Secondly, there is a general consensus that that a mammogram is of high quality when it enables the radiologist to discern the presence or absence of the mammographic features of breast cancer in the image with high sensitivity and specificity [32], and when it yields adequate diagnostic information with the least possible radiation exposure to the breast $[33,34]$. These requirements are particularly needed when screening women with breast implants. This is the reason why the endpoints of this study encompassed both the screening and management indicators and some essential dosimetric parameters.

\section{Comparison with the literature}

We are not aware of previous publications reporting data comparable with ours. However, protocols for the management of women with breast implants in the screening setting 
Table 5 Average glandular dose (AGD) and for BT (breast thickness) for the four views according to different machines and Eklund technique

\begin{tabular}{|c|c|c|c|c|c|c|c|c|c|c|c|}
\hline \multicolumn{6}{|l|}{ 2000/ED } & \multicolumn{6}{|l|}{ Selenia } \\
\hline \multirow[b]{2}{*}{ Eklund (mm) } & & \multicolumn{2}{|l|}{$\mathrm{RCC}$} & \multicolumn{2}{|l|}{ LCC } & \multirow[b]{2}{*}{ Eklund } & & \multicolumn{2}{|l|}{$\mathrm{RCC}$} & \multicolumn{2}{|l|}{ LCC } \\
\hline & & AGD (mGy) & $\mathrm{BT}(\mathrm{mm})$ & AGD (mGy) & $\mathrm{BT}(\mathrm{mm})$ & & & AGD (mGy) & $\mathrm{BT}(\mathrm{mm})$ & AGD (mGy) & BT \\
\hline \multirow[t]{3}{*}{ Yes } & Mean & 1.5 & 43.7 & 1.6 & 43 & \multirow[t]{3}{*}{ Yes } & Mean & 1 & 41.2 & 0.8 & 40 \\
\hline & Median & 1.5 & 45 & 1.6 & 45 & & Median & 0.9 & 40 & 0.7 & 39 \\
\hline & Range & $0.7-4.8$ & $18-80$ & $0.7-2.7$ & $20-75$ & & Range & $0.6-4$ & $21-68$ & $0.5-2$ & $23-70$ \\
\hline \multirow[t]{3}{*}{ No } & Mean & 1.3 & 61.6 & 1.3 & 61.2 & \multirow[t]{3}{*}{ No } & Mean & 1.4 & 79 & 1 & 81 \\
\hline & Median & 1.2 & 56 & 1.2 & 54 & & Median & 1 & 79 & 0.9 & 90 \\
\hline & Range & $0.8-7.7$ & $37-105$ & $0.7-2.7$ & $31-127$ & & Range & $0.8-6.2$ & $43-108$ & $0.9-1.6$ & $44-100$ \\
\hline
\end{tabular}

RCC, Right Craniocaudal; LCC, Left Craniocaudal; RMLO, Right Medial-Lateral Oblique; LMLO, Left Medial-Lateral Oblique

are operating in other countries including, to our knowledge, Australia [35], UK [17] and Spain (Cantabric regional programme) [36]. All three protocols include dedicating additional time to these women and performing mammography in dedicated screening slots. Additional time is required because of the need to collect an accurate anamnesis about the type of prosthesis and its state [35] and to perform all required manoeuvres [17, 35]. The Spanish and the British protocols also point out that the mammogram is not aimed at assessing the state of the prosthesis, but just at early detection of breast cancer $[17,36]$. The UK protocol emphasizes the radiographers' training needs. In the NHS Breast Screening Programme (NHSBSP), indeed, all radiographers undertaking the Eklund technique should give proof that they have been educated and trained to perform the manoeuvre [17]. None of the protocols mentioned above include performing further tests, apart from those already needed for the workup of suspicious mammograms.

What our protocol has in common with the models from Australia, UK, and Spain is that it reserves dedicated slots for women with breast implants and that the radiographic staff are trained in the Eklund technique. Also the American Cancer Society, too, highlights the importance of these practices, in order to guarantee a correct execution of the implant displacement views [37].

\section{Study limitations}

This study had two major limitations that need to be corrected in future investigations. Firstly, our protocol is the only one offering additional non-invasive tests (US and particularly $\mathrm{CBE}$ ), in order to overcome any detection impairment due to the prosthesis. Because of its poor statistical power, however, the study was not designed to assess the usefulness of adding other tests to mammography in order to increase the detection rate, nor to show whether these actions lead to unintended harms.
Secondly, prostheses implanted in Europe are usually silicone gel-filled and textured [3] and, in most cases, their location is partially submuscular [38]. A recent survey has shown that, elsewhere, the most common materials and locations may differ from to the ones observed in our cohort. Consequently, our results cannot be generalized to settings where prostheses have different characteristics.

In addition, we specify that the collection of dosimetric parameters was performed only on a representative sample, not the total number of images, for reason of feasibility.

\section{Conclusions}

To our knowledge, this study is the first to report screening indicators and dosimetric parameters collected in sessions of a BSP specifically dedicated to augmented women in Italy and elsewhere. The ever-increasing prevalence of this condition will set a challenge to BSPs for the next future, both from the technical and the diagnostic perspective. In this respect, our work could represent a starting point to plan the necessary resources, when integrating screening programs for augmented women.

Despite the limitations of this study, our experience shows that the management of these patients is feasible and can improve over time-provided that they are referred to a specialized centre where the staff is properly trained and the mammography system is up-to-date and subjected to quality control programme.

Author contributions Silvia Deandrea, Silvana Castaldi, Maria Silvia Sfondrini, Stella Pedilarco, Mauro Campoleoni, Ester Luconi, Niccolò Principi, and Laura Cavazzana were responsible for conceptualization. Silvia Deandrea, Laura Cavazzana, Niccolò Principi, Anan Judina Bastiampillai, Lucia Bracchi, Antonio Piscitelli were responsible for data curation. Silvia Deandrea and Silvana Castaldi were responsible for project administration. Silvia Deandrea, Mauro Campoleoni, Stella Pedilarco, Silvana Castaldi and Maria Silvia Sfondrini were responsible 
for investigation. Silvia Deandrea, Laura Cavazzana, Niccolò Principi, Ester Luconi, Mauro Campoleoni were responsible for writing-original draft. Laura Cavazzana and Niccolò Principi were responsible for resources. Ester Luconi was responsible for formal analysis and methodology. Anan Judina Bastiampillai, Lucia Bracchi, Lauro Bucchi, Silvana Castaldi, Anna Rita Silvestri, Maria Silvia Sfondrini, Antonio Piscitelli and Stella Pedilarco were responsible for writing-review $\&$ editing. Anna Rita Silvestri and Silvana Castaldi were responsible for supervision.

Funding Open access funding provided by Università degli Studi di Milano within the CRUI-CARE Agreement. No funds, grants, or other support was received.

Availability of data and material Data available on request from the authors.

Code availability Code available on request from the authors.

\section{Declarations}

Conflicts of interest The authors have no relevant financial or nonfinancial interests to disclose.

Ethical approval Institutional Review Board approval was not required according to the regional law 24.12.2012 n.3 of Lombardy region, Italy.

Informed consent Written informed consent was not required for this study according to the regional law 24.12.2012 n.3 of Lombardy region, Italy.

Open Access This article is licensed under a Creative Commons Attribution 4.0 International License, which permits use, sharing, adaptation, distribution and reproduction in any medium or format, as long as you give appropriate credit to the original author(s) and the source, provide a link to the Creative Commons licence, and indicate if changes were made. The images or other third party material in this article are included in the article's Creative Commons licence, unless indicated otherwise in a credit line to the material. If material is not included in the article's Creative Commons licence and your intended use is not permitted by statutory regulation or exceeds the permitted use, you will need to obtain permission directly from the copyright holder. To view a copy of this licence, visit http://creativecommons.org/licenses/by/4.0/.

\section{References}

1. U.S. Food and Drug Administration Center for Devices and Radiological Health (2011) FDA update on the safety of silicone gelfilled breast implants. U.S. food and drug administration center for devices and radiological health, Silver Spring MD

2. American Society for Aesthetic Plastic Surgery (2017) Cosmetic surgery national data bank statistics. Aesthet Surg J 37:1-29

3. International Society For Aesthetic Plastic Surgery (2018) International survey on aesthetic/cosmetic procedures performed in 2017. International society for aesthetic plastic surgery, Hanover

4. Hoshaw SJ, Klein PJ, Clark BD et al (2001) Breast implants and cancer: causation, delayed detection, and survival. Plast Reconstr Surg 107:1393-1407
5. Tuli R, Flynn RA, Brill KL et al (2006) Diagnosis, treatment, and management of breast cancer in previously augmented women. Breast J 12:343-348

6. Noels EC, Lapid O, Lindeman JH et al (2015) Breast implants and the risk of breast cancer: a meta-analysis of cohort studies. Aesthet Surg J 35(1):55-62

7. Leberfinger AN, Behar BJ, Williams NC et al (2017) Breast implant-associated anaplastic large cell lymphoma: a systematic review. JAMA Surg 152(12):1161-1168

8. Smalley S (2003) Breast implants and breast cancer screening. J Midwifery Womens Health 48:329-337

9. Miglioretti DL, Rutter CM, Geller BM et al (2004) Effect of breast augmentation on the accuracy of mammography and cancer characteristics. JAMA 291:442-450

10. Skinner KA, Silberman H, Dougherty W et al (2001) Breast cancer after augmentation mammoplasty. Ann Surg Oncol 8:138-144

11. McIntosh SA, Horgan K (2008) Augmentation mammoplasty: effect on diagnosis of breast cancer. J Plast Reconstr Aesthet Surg 61(2):124-129

12. Lavigne E, Holowaty EJ, Pan SY et al (2013) Breast cancer detection and survival among women with cosmetic breast implants: systematic review and meta-analysis of observational studies. BMJ 346:f2399

13. Hölmich LR, Mellemkjaer L, Gunnarsdóttir KA et al (2003) Stage of breast cancer at diagnosis among women with cosmetic breast implants. Br J Cancer 88(6):832-838

14. Cho EH, Shammas RL, Phillips BT et al (2017) Breast cancer after augmentation: oncologic and reconstructive considerations among women undergoing mastectomy. Plast Reconstr Surg 139(6):1240-9

15. Silverstein MJ, Handel N, Gamagami P et al (1990) Mammographic measurements before and after augmentation mammaplasty. Plast Reconstr Surg 86(6):1126-1130

16. Azzi AJ, Gornitsky J, Viezel-Mathieu A et al (2018) The impact of implant location on breast cancer characteristics in previously augmented patients: a systematic literature analysis. J Cancer Prev 23(2):93-98

17. Public Health England (2017) NHS breast screening program. Screening women with breast implants. Public Health England, London

18. Handel N (2007) The effect of silicone implants on the diagnosis, prognosis, and treatment of breast cancer. Plast Reconstr Surg 120:81S-93S

19. Perry N, Broeders M, deWolf C et al (2006) European guidelines for quality assurance in breast cancer screening and diagnosis. European Breast Cancer Network (EBCN), Lyon

20. Sardanelli F, Fallenberg EM, Clauser P et al (2017) Mammography: an update of the EUSOBI recommendations on information for women. Insights Imaging 8(1):11-18

21. Green LA, Karow JA, Toman JE et al (2018) Review of breast augmentation and reconstruction for the radiologist with emphasis on MRI. Clin Imaging 47:101-117

22. Eklund GW, Busby RC, Miller SH et al (1988) Improved imaging of the augmented breast. AJR Am J Roentgenol 151:469-473

23. Soares Couto L, Freitas-Junior R, Silveira Correa R et al (2019) Mean glandular dose in digital mamography in women with breast implants. J Radiol Prot 39(2):498-510

24. Regione Lombardia, DG Welfare (2019) Gli screening oncologici in Lombardia. Rapporto 2019. Regione Lombardia, DG Welfare, Milan

25. Osservatorio nazionale screening (2018) Rapporto Ons 2018

26. Legge Regionale 11 agosto 2015, n. 23 Evoluzione del sistema sociosanitario lombardo: modifiche al Titolo I e al Titolo II della legge regionale 30 dicembre 2009, n. 33 (Testo unico delle leggi regionali in materia di sanità) 
27. IRCCS Ospedale Maggiore Policlinico Ca' Granda Foundation (2014) Carta dei Servizi. IRCCS Ospedale Maggiore Policlinico Ca' Granda Foundation, Milan

28. Ospedale Maggiore Policlinico Ca' Granda Foundation. Direzioni Generale, Sanitaria e Amministrativa (a cura di), (2015) "Relazione di fine mandato". IRCCS Ospedale Maggiore Policlinico Ca' Granda Foundation, Milan

29. van Engen R, van Woudenberg S, Bosmans H et al (2013) European protocol for the quality control of the physical and technical aspects of mammography screening. Fourth Edition-Supplements. EUREF, European Reference Organisation for Quality Assured Breast Screening and Diagnostic Services, Nijmegen

30. KoNstanzInformation MinEr, KNIME.AG

31. R Core Team (2017) R: A language and environment for statistical computing. R Foundation for Statistical Computing, Vienna, Austria

32. Caldwell CB, Fishell EK, Jong RA et al (1992) Evaluation of mammographic image quality: pilot study comparing five methods. AJR Am J Roentgenol 159:295-301

33. Reis A, Pascoal A, Sakellaris T et al (2013) Quality assurance and quality control in mammography: a review of available guidance worldwide. Insights Imaging 4:539-553
34. Ng KH, Jamal N, DeWerd L (2006) Global quality control perspective for the physical and technical aspects of screen-film mammography: image quality and radiation dose. Radiat Prot Dosimetry 121:445-451

35. The Royal Australian and New Zealand College of Radiologists (2002) Breast imaging: a guide for practice. The Royal Australian and New Zealand College of Radiologists, Sydney

36. Sánchez Movellán M, Vega Bolivar A, Ortega García E et al (2013) Protocolo para el manejo radiológico de las mujeres con protésis mamarias. Gobierno de Cantabria, Consejeria de sanidad y servicios sociales, Dirección General de Salud Pública, Santander

37. American Cancer Society (2019) Mammograms for women with breast implants. American Cancer Society, Atlanta GA

38. Heidekrueger PI, Sinno S, Hidalgo DA et al (2018) AesthetSurg J 38(2):133-148

Publisher's Note Springer Nature remains neutral with regard to jurisdictional claims in published maps and institutional affiliations. 